every group of the fourth class, of order $p^{5}$, where $p>3$, that has an operator of order $p^{2}$ is generated by an operator of order $p^{2}$ and three of order $p .^{*}$ No such group can be a group of cogredient isomorphisms. Therefore :

$A$ group of order $p^{m}$, where $p$ is a prime $>3$, that contains an operator of order $p^{m-3}$ is of class $k$, where $k \equiv 4$.

CoRnell Univeritity.

\title{
PROOF THAT THE GROUP OF AN IRREDUCIBLE LINEAR DIFFERENTIAL EQUATION IS TRANSITIVE.
}

BY DR. SAUL EPSTEEN.

(Read before the American Mathematical Society, December 28, 1901.)

We will define with Frobenius $\dagger$ the linear differential equation

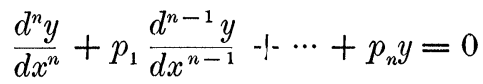

to be irreducible when it has no integral in common with a differential equation of the same character, but of a lower order.

Picard has shown $\$$ that for the equation (1) a linear homogeneous group

$$
Y_{i}=\sum_{k} a_{i k} y_{k}
$$

plays the same rôle as the group of substitutions plays in the Galois theory of algebraic equations.

It is the object of this brief paper to show that the group (2) of the equation (1) will be transitive $\S$ when the equation is irreducible and intransitive when the equation is reducible, and we shall carry the proof out on lines analogous to the corresponding theorem in algebra.||

The basis of our proof is the Lagrange-Vessiot theorem, that if the rational differential function $S(y)$ of the in-

\footnotetext{
* Bagnera, Ann. di Matematicn, ser. 3, vol. 1 (1898), p. 218.

† Frobenius, Crelle, vol. 76.

I Picard, Comptes rendus, 1883.

\&ie-Eugel, Transformationsgruppen, I., ch. 13.

Netto, Substitutionstheorie, § 154 .

E. Vessiot, Annales de l'Ec. Norm. Sup., 1892.
} 
tegral is invariant under the group of the differential function $R(y)$, then $S$ can be expressed rationally by means of $R$, the coefficients of the equation (1), their derivatives, and $x$, that is : $S=$ Rational Function $\left(R, R^{\prime}, \cdots, p_{1}, \cdots, p_{n}\right.$, $\left.p_{1}{ }^{\prime}, \cdots, p_{n}{ }^{\prime}, x\right)$.

Suppose that the group of the equation (1) is intransitive, or in other words that we can transform the system of integrals

$$
Y_{1}, \cdots, Y_{m} \quad \text { into } \quad y_{1}, \cdots, y_{m} \quad(m<n)
$$

by means of the group, but not

$$
Y_{m+1}, \cdots, Y_{n} \text { into } y_{m+1}, \cdots, y_{n} .
$$

Let us now form the linear differential equation of the $m$ th order which has $Y_{1}, \cdots, Y_{m}$ as a fundamental system of integrals

$$
\frac{d^{m} y}{d x^{m}}+q_{1} \frac{d^{m-1} y}{d x^{m-1}}+\cdots+q_{n} y=0 .
$$

The coefficients of this equation in terms of the integrals are

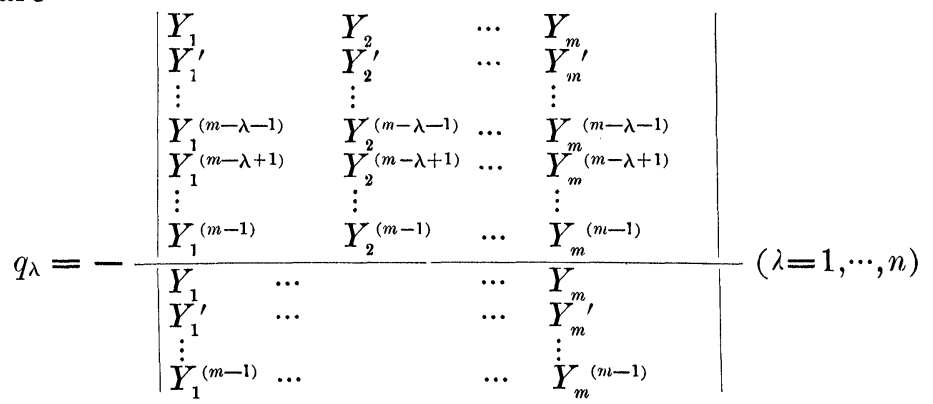

Our hypothesis is that the group (2) will, by a suitable choice of the parameters, bring the system of solutions $Y_{1}, \cdots, Y_{m}$ exactly into $y_{1}, \cdots, y_{m}$; that is to say, the coefficients $q$ of the equation (3) are invariant under the group of (1) and consequently, by the Lagrange-Vessiot theorem,

$$
q_{\chi}=\text { Rat. Funct. }\left(p_{1}, \cdots, p_{n}, p_{1}{ }^{\prime} \cdots, p_{n}{ }^{\prime}, x\right) \text {. }
$$

The equation (1) has therefore the $m$ integrals $Y_{1}, \ldots, Y_{m}$ $(m<n)$ in common with the equation (3). But the equation (3) is linear of lower order than (1), and. as we have 
just seen, its coefficients are rational. It follows from the definition that the equation ( 1 ) is reducible.

Conversely, it is now easy to see that if the equation (1) is reducible, its group must be intransitive.

We are therefore enabled to enunciate the following theorem, exactly analogous to a theorem in algebra :

The group of an irreducible linear differential equation is transitive.

GöTTINGEN, INecember, 1901.

\section{LINES OF LENGTH ZERO ON SURFACES.}

BY DR. L. P. EISENHART.

(Read before the American Mathematical Society, December 28, 1901.)

By definition, the directions of the double system of lines of length zero on a surface are given by equating to zero the expression for the square of the linear element of the given surface, that is, in the Gauss notation,

$$
E d u^{2}+2 F d u d v+G d v^{2}=0 .
$$

Since these lines are always imaginary and since the lines of curvature of a real surface are always real there is no real surface whose lines of curvature are of length zero. It is well known that lines of length zero form a conjugate system upon a minimal surface and further that this is a characteristic property of such surfaces. In order that asymptotic lines be of length zero it is necessary and sufficient that the two fundamental forms of the surface be proportional. Hence the sphere is the only real surface whose asymptotic lines are of length zero ; they are the imaginary rectilinear generatrices of the sphere. When the parameters of these lines are taken as conjugate imaginaries and the sphere is of radius unity and with center at the origin, the cartesian coördinates have the following expressions :

$$
x=\frac{u+v}{1+u v}, \quad y=i \frac{v-u}{1+u v}, \quad z=\frac{u v-1}{1+u v} . *
$$

We propose now the following problem :

\footnotetext{
* Darboux, Leçons, I, p. 245.
} 